\title{
Elemental Abundances of Ultra-Heavy GCRs measured by SuperTIGER and ACE-CRIS and the Origin of Galactic Cosmic Rays
}

\author{
T. Hams ${ }^{* 1,6}$, W.R. Binns ${ }^{2}$, T.J. Brandt ${ }^{1}$, E.R. Christian ${ }^{1}$, A.C. Cummings ${ }^{3}$, \\ G.A. de Nolfo ${ }^{1}$, P.F. Dowkontt ${ }^{2}$, M.H. Israel ${ }^{2}$, J.F. Krizmanic ${ }^{1,6}$, A.W. Labrador ${ }^{3}$, \\ R.A. Leske ${ }^{3}$, J.T. Link ${ }^{1,6}$, R.A. Mewaldt ${ }^{3}$, J.W. Mitchell ${ }^{1}$, R.P. Murphy ${ }^{2}$, B.F. Rauch ${ }^{2}$, \\ K. Sakai ${ }^{1,6}$, M. Sasaki ${ }^{1,6}$, E.C. Stone ${ }^{3}$, T.T. von Rosenvinge ${ }^{1}$, C.J. Waddington ${ }^{4}$, \\ J.E. Ward ${ }^{2} ;$ A.J. Westphal ${ }^{7}$, M.E. Wiedenbeck ${ }^{5}$ \\ ${ }^{1}$ NASA Goddard Space Flight Center, Greenbelt, MD 20771, USA \\ ${ }^{2}$ Washington University, St. Louis, MO 63130, USA \\ ${ }^{3}$ California Institute of Technology, Pasadena, CA 91125, USA \\ ${ }^{4}$ University of Minnesota, Minneapolis, MN 55455, USA \\ ${ }^{5}$ Jet Propulsion Laboratory, California Institute of Technology, Pasadena, CA 91109, USA \\ ${ }^{6}$ Center for Research and Exploration in Space Science and Technology (CRESST) \\ ${ }^{7}$ University of California, Berkeley, CA, 94720, USA
}

E-mail: Thomas. Hams @nasa.gov

The Super Trans-Iron Galactic Element Recorder (SuperTIGER) long-duration balloon instrument and the Cosmic Ray Isotope Spectrometer (CRIS) on the NASA Advanced Composition Explorer (ACE) satellite have measured the abundances of galactic cosmic-ray elements from ${ }_{10} \mathrm{Ne}$ to ${ }_{40} \mathrm{Zr}$ with high statistics and single-element resolution. SuperTIGER launched from Williams Field, McMurdo Station, Antarctica, on December 8, 2012, flying for a record 55 days. During that flight we detected $\sim 1,300$ nuclei with atomic number $Z \geq 30$. The resolution in charge (Z) of SuperTIGER is excellent, with $\sigma_{Z} \approx 0.16$ c.u. at ${ }_{26} \mathrm{Fe}$. SuperTIGER is sensitive to nuclei with energy at the top of the atmosphere of $E>0.8 \mathrm{GeV} /$ nucleon. The instrument has now been recovered and preparations are underway for its next flight. ACE/CRIS has been taking data in space for more than 17 years since launch in 1997, has collected $\sim 625$ nuclei with atomic number $Z \geq 30$, and shows excellent resolution with clear separation between the charges for $30 \leq Z \leq 40$. $\mathrm{ACE} / \mathrm{CRIS}$ is sensitive to nuclei in the energy range $150 \leq E \leq 600 \mathrm{MeV} /$ nucleon. Preliminary results from the balloon-borne SuperTIGER show good agreement with ACE measurements in space, validating our corrections to SuperTIGER abundances for nuclear interactions in the atmosphere. The results from these experiments will be discussed in the context of the OB association model for the origin of galactic cosmic rays. Future missions to measure elemental abundances to higher Z, the SuperTIGER-II LDB instrument and the orbiting Heavy Nuclei eXplorer (HNX) mission, will also be discussed.

The 34th International Cosmic Ray Conference,

30 July- 6 August, 2015

The Hague, The Netherlands 


\section{Introduction}

The origin of galactic cosmic-ray (GCR) nuclei is one of the great open questions in astrophysics. Direct measurements of the elemental and isotopic abundances with balloon- and spacebased instruments have been used to constrain and test theories on the origin of cosmic rays. Recent results of the elemental composition of ultra-heavy galactic cosmic-ray (UHGCR) nuclei from the TIGER[1] and SuperTIGER balloon instrument and the CRIS instrument onboard the ACE spacecraft support the emerging model that GCRs originate in OB associations.

The SuperTIGER (Super Trans-Iron Galactic Element Recorder) instrument, a long-duration balloon payload, was developed to measure the elemental composition of UHGCR nuclei in the charge range $26 \leq Z \leq 40$ with high statistical precision and excellent charge resolution, as well as provide exploratory measurements into the $40<Z \leq 60$ range. A secondary objective was to accurately measure the energy spectra of the more abundant light elements with $12 \leq Z \leq 30$ in the energy range $\sim 0.8-10 \mathrm{GeV} /$ nucleon. SuperTIGER was flown during the $2012 / 2013$ Austral Summer, returning data on over $50 \times 10^{6}$ GCR nuclei with $Z \geq 10$, including $\sim 1,300$ with $Z>29$, and $\sim 60$ with $Z>49$ in 55 days at float.

Based on the observed enrichment of elements in cosmic rays with low First Ionization Potential (FIP), Cassé \& Goret[2] and Meyer[3] suggested that the source of cosmic rays was the coronae of stars. According to Schmelz et al.[4], the fractionation in particles from the Sun "probably results from a separation of ions and neutrals, which takes place between the photosphere and the corona at temperatures of $6000-10,000 \mathrm{~K}$." The coronal material would then be injected into the interplanetary medium by stellar winds or flare events and later accelerated to cosmic-ray energies by shocks from nearby supernovae. In 1989, a paper by Binns et al.[5], giving the results of the HEAO-3-C3 experiment concluded, "These abundances are consistent with a cosmic-ray source having a composition similar to that of the solar system, but subject to source fractionation correlated with the first ionization potential (FIP) of each element".

However, Epstein[6] and Bibring \& Cesarsky[7] showed that refractory elements existing in interstellar dust grains should be preferentially accelerated, compared to elements existing primarily in the gas phase, owing to their large mass-to-charge ratio. The relative enrichment of refractory elements should be similar to the FIP enrichment since many low-FIP elements are refractory and most high-FIP elements are volatile. This work was later extended in a pair of papers Meyer et al.[8] and Ellison et al.[9], which found that the dust grains (refractories) themselves are accelerated, material is sputtered off and then the sputtered ions are reaccelerated to cosmic-ray energies. Additionally, their model predicted that the abundances of the volatile elements relative to iron should be mass dependent, but refractory abundances should be mass independent.

Soon after that, Westphal et al.[10] published measurements of the $\mathrm{Z}=70-92$ charge range from the Trek instrument aboard the Russian space station Mir, which resolved even- $Z$ nuclei in this charge range for the first time. They concluded that the measured relative abundances for $Z=74$ through 82 were inconsistent with a chromospheric origin, but consistent with an origin in interstellar gas and dust, with refractory elements preferentially accelerated.

\footnotetext{
${ }^{*}$ Speaker.

${ }^{\dagger}$ Now at Institut de Fisica d'Altes Energies (IFAE), Ballaterra, Spain
} 
More recently, abundances measured by TIGER at GeV/nucleon energies [1], ACE at energies of hundreds of $\mathrm{MeV} /$ nucleon [11], and CREAM at TeV/nucleon energies [12], show that refractory elements are enriched by a factor of about four over volatiles[1, 11]. Furthermore, this enrichment is mass dependent with similar slopes for both refractories and volatiles, and the ordering of these element abundances with atomic mass is greatly improved by comparing them to a mix of normal interstellar material (with solar system abundances) with massive star outflow and ejecta [13], rather than normal interstellar material alone.

The picture that is currently widely accepted is that a large fraction of cosmic rays originate in OB associations. The OB association origin of cosmic rays was first discussed by Montmerle[14] and Cesarsky \& Montmerle[15]. More recently Higdon et al.[16] and Higdon \& Lingenfelter $[17,18,19]$ developed this model in more detail. In this picture cosmic-ray nuclei originate primarily within OB associations, concentrations of young, short-lived, massive stars that form superbubbles in the interstellar medium by the combination of their stellar winds and supernova shocks. The accelerators are believed to be supernova shocks, shocks from the winds of the massive precursor stars [16, 17, 18, 20], and colliding winds of massive binaries [21]. Cosmic rays are then accelerated from a mixture of old interstellar gas and dust (with solar-system composition; e.g., [22]), the wind outflow from massive stars (including Wolf-Rayet stars and their precursor phases), and ejecta from core-collapse supernovae (SNII, SNIb,c). The measured composition of cosmicray isotopes and elements was found to be consistent with a mix of interstellar material with outflow and ejecta from massive stars [23, 1], and with refractory elements enhanced by about a factor of four over volatile elements. Prantzos[24], on the other hand, can not quantitatively account for the measured ${ }^{22} \mathrm{Ne} /{ }^{20} \mathrm{Ne}$ ratio [23] in his model for an $\mathrm{OB}$ association origin.

The TIGER balloon payload flew in 2001 and 2003 and measured the more abundant individual elements up to ${ }_{38} \mathrm{Sr}$. The SuperTIGER flight significantly improved the statistics of these nuclei, extended statistically significant measurements up to at least ${ }_{40} \mathrm{Zr}$, and sampled the $\mathrm{Z}=50$ 60 charge range. Sensitive measurements of these rare $Z \geq 30$ elements by SuperTIGER will enable us to test the $\mathrm{OB}$ association/superbubble origin model in more detail by reducing the uncertainties and extending the reach in mass range of the refractory and volatile elements to further explore preferential acceleration and its mass dependence for ultra-heavy cosmic-ray nuclei. We will also search for possible enrichments from nucleosynthesis in massive stars (Woosley \& Heger[13]).

As mentioned above, SuperTIGER also measures the energy spectra of the more abundant elements. Heinz and Sunyaev[25] have shown that nearby microquasars may impose narrow features in the energy spectra of cosmic-ray nuclei in the energy range from $\sim 1-10 \mathrm{GeV} /$ nucleon. The complement of sensors used in SuperTIGER allows us to measure with greater precision than before (Geier et al.[26]) the energy spectra of GCR nuclei up through ${ }_{30} \mathrm{Zn}$ over the energy range of $\sim 0.8$ to $10 \mathrm{GeV} /$ nucleon (top of the atmosphere energies).

\section{SuperTIGER}

\subsection{Instrument}

The SuperTIGER balloon payload consists of two identical modules, each comprised of scintillating fiber hodoscopes (H1, H2), three scintillator detectors (S1, S2, and S3), and an aerogel 
and an acrylic Cherenkov detector (C0, C1)[27] (Fig. 1 and 2). The hodoscope determines the trajectory of incident particles for mapping and angle corrections. The scintillators and Cherenkov detectors determine the particle charge and energy. A detailed instrument discussion can be found in[28] and references therein.
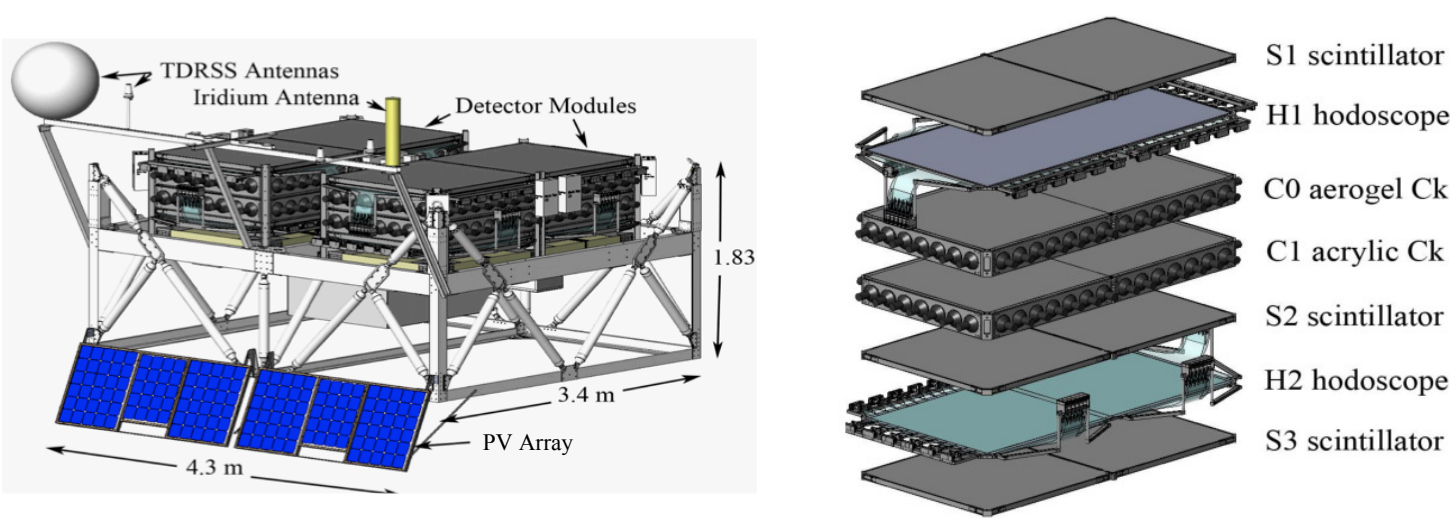

Figure 1: The SuperTIGER instrument.

Figure 2: Single module shown in expanded view.

The hodoscopes use a coded readout scheme originally developed for TIGER to limit the number of PMTs required. Each hodoscope plane uses two orthogonal layers of square scintillating fibers covering a fiducial area of $2.4 \mathrm{~m} \times 1.16 \mathrm{~m}$. A good hodoscope event has a hit at both coarse and fine ends, resulting in a position uncertainty of $2.3 \mathrm{~mm}[28]$. The PMT signals are pulseheight analyzed so that large signals from heavy nuclei can be readily distinguished from knock-on electrons. In an effort to reduce unwanted nuclear interactions in the detector support material traversed by the particles, GSFC developed an ultra-low density Al-foam composite, reducing the nuclear interactions by $\sim 20 \%$ compared to the materials used in TIGER.

Each scintillation counter layer was split on the long axis into two equal size half-modules. Each half-module scintillation detector uses a single piece of ELJEN Technology EJ-208B plastic scintillator $(1.16 \mathrm{~m} \times 1.16 \mathrm{~m} \times 1 \mathrm{~cm})$. The scintillation light produced is carried to the edges of the sheet by total internal reflection and is coupled through a thin air gap to four wavelength-shifter (WLS) bars around its perimeter. Each WLS bar is viewed at both ends by $2.54 \mathrm{~cm}$ diameter PMTs. The scintillator material and the WLS bars were wrapped in a thin, highly-reflective aluminized kapton film to decouple the internal reflection surfaces from the support substrate and improve light collection and uniformity. The PMT bases used radically tapered voltage dividers providing nearly linear response up to $2 \times 10^{5}$ photoelectrons (p.e.) and an effective dynamic range of $2 \times 10^{4}$. In addition to providing $d E / d x$ measurements, the scintillation counter layers generated the primary event trigger.

The Cherenkov detectors for SuperTIGER were based on the extensive experience of the collaboration with silica-aerogel and acrylic Cherenkov detectors. Light integration volumes lined with highly reflective GORE DRP Light-Reflective Material of $0.25 \mathrm{~mm}$ thickness collects the Cherenkov light produced by the silica-aerogel $(\mathrm{C} 0)$ and acrylic $(\mathrm{C} 1)$ radiators. Each optical volume has an aperture of $118 \mathrm{~cm} \times 240 \mathrm{~cm}$ and is $20 \mathrm{~cm}$ tall. The enclosures are divided into two half-module structures for recovery, but these are open at one end and so create a unified optical 
system. Each detector is read out by $4212.7 \mathrm{~cm}$ high quantum efficiency PMTs. Each C0 module contains eight aerogel blocks, each approximately $55 \mathrm{~cm} \times 55 \mathrm{~cm}$ and $3 \mathrm{~cm}$ tall. Three of the four half-modules contain aerogel blocks with $\mathrm{n}=1.043$ (12 blocks total), while one half-module contains four blocks with $\mathrm{n}=1.025$. These have thresholds of 2.5 and $3.3 \mathrm{GeV} /$ nucleon, respectively. The $\mathrm{C} 1 \mathrm{Cherenkov}$ counter uses acrylic radiators with bis-MSB wavelength shifter added ( $25 \mathrm{mg} /$ liter doping). This material has an index of refraction of 1.49, corresponding to a threshold of $0.35 \mathrm{GeV} /$ nucleon. In each module the radiator consists of two $1.16 \mathrm{~m} \times 1.16 \mathrm{~m}$ sheets. The Cherenkov detector PMTs were mounted in custom hydroformed $0.3 \mathrm{~mm}$ thick mu-metal sleeves that served as magnetic shields, mechanical support and light close-out. Their bases used voltage dividers that were even more radically tapered than those for the scintillators, and which could measure from 10 to $\sim 2 \times 10^{5}$ pe (dynamic range of $2 \times 10^{4}$ ) with only $2 \%$ non-linearity in order to span the $10 \leq \mathrm{Z} \leq 60$ range.

\subsection{Flight}

SuperTIGER was launched on Dec. 8, 2012 (UTC), from Williams Field near McMurdo Station on Ross Island, Antarctica. The payload flew for over 55 days at altitudes from about $35.2 \mathrm{~km}$ to $39.7 \mathrm{~km}$ corresponding to a residual atmosphere of $\sim 6.4$ to $3.5 \mathrm{~g} / \mathrm{cm}^{2}$, respectively (see [28]). After about 2.7 polar circumnavigations, the flight was terminated on Feb. 1, 2013 due to concerns about increasing instability in the high-altitude winds. The payload termination sequence was carried out "over-the-horizon" and the landing site was $82^{\circ} 14^{\prime} 44.40^{\prime \prime}$ South latitude and $81^{\circ} 54^{\prime} 40.80^{\prime \prime}$ West longitude on the West Antarctic Ice Sheet (WAIS), $1625 \mathrm{~km}$ from McMurdo and $600 \mathrm{~km}$ from the nearest long-term US camp (WAIS Divide). The flight set duration records for heavy scientific payloads and for heavy-lift scientific balloons.

In-flight the instrument performed nominally and returned excellent data on over $50 \times 10^{6}$ cosmic-ray nuclei above the trigger threshold at $\sim Z \geq 10$. Nearly all high-priority data $(Z \geq$ 22) could be sent through the NASA Tracking and Data Relay Satellite System (TDRSS) when satellite view angles supported transmission rates of $20 \mathrm{kbs}$ and higher. Large fractions of lower priority data $(10 \leq Z \leq 22)$ were also returned. Overall, considering outages and very-low-rate periods, TDRSS data transmission was about $80 \%$ efficient, giving the equivalent of 44 days of data recorded for high-Z events. All triggered events occurring during the two LOS (line-of-sight) periods at the beginning of the flight and the end of the first orbit/beginning of the second, were returned.

\subsection{Recovery}

As a result of the very long balloon flight the termination occurred late in the 2012/2013 Antarctic season. Furthermore, the payload location was out of range for available aircraft from McMurdo and consequently not even a payload overflight of the landing site was possible at the time.

During the subsequent 2013/2014 season, a recovery attempt was mounted and the recovery team reached South Pole Station. Due to a string of bad weather conditions and limited aircraft time, only one flight from South Pole Station (via deep field fuel cache) to the payload was possible. The SuperTIGER landing area is subject to persistent ground winds and snow drift. That season 
the snow surface was too rough to land an aircraft safely and only a brief overflight was possible, which revealed that the payload had turned upside down and the detector systems were buried in the snow with only the gondola parts visible.

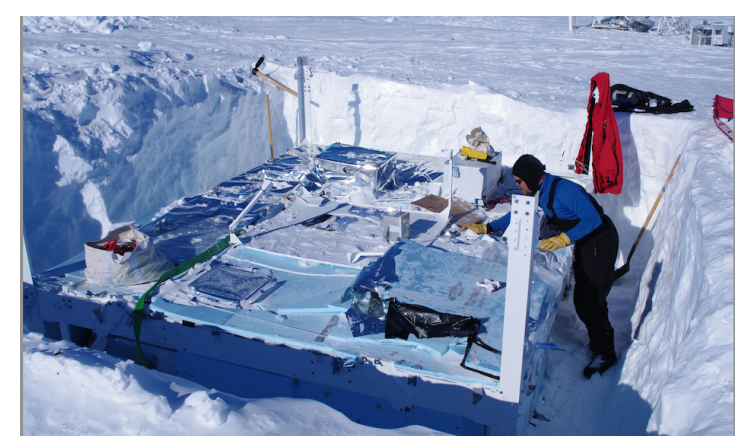

Figure 3: The SuperTIGER instrument turned upside-down and was buried under 2 meter of snow.

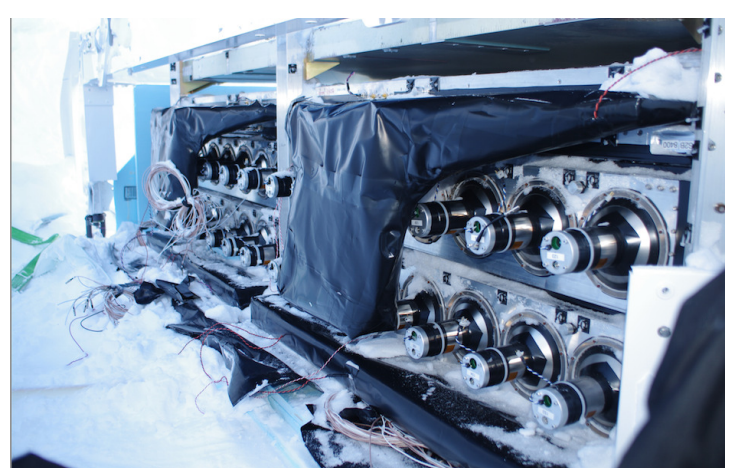

Figure 4: Site view of one detector module after the electronics box, HV cables, and signal cables were carefully removed.

A complete recovery was successfully carried out in the 2014/2015 Austral Summer, two years after the flight. A long-range, heavy-lift Basler aircraft (DC-3 turboprop conversion) was assigned to extract the payload, support equipment and personnel from the SuperTIGER site to an established US camp with heavy cargo-handeling capability. The Basler is a tail-dragging aircraft and required a groomed skiway for take off and landing. The recovery plan had the 3 person skiway groom team plus one SuperTIGER science team member deployed to the recovery site. They reached the site on Dec. 28, 2014 established camp, started with the skiway construction, and begun digging out the payload, which by now was buried up to 2 meters in snow (Fig. 3).

After completion of the skiway, estimated to take about 5-7 days, the remaining 2 SuperTIGER team members and the bulk recovery tools were expected to stage through South Pole Station and reach the recovery site by Basler. Due to the delay of the second flight, the initial recovery plan could not be executed. The abandoned plan foresaw that the 3 person recovery team would camp at the site for a few days, where they would disassemble the instrument and prepare the components for transportation to the South Pole Station by Basler.

Due to weather delays the remaining 2 SuperTIGER recovery team members were not able to reach the site until Jan. 24, 2015, about 3 weeks later than anticipated. By that time the initial team had used basic tools at their disposal and already dug out the payload, carefully removed all the electronic boxes and cables (Fig. 4), disassembled and staged the gondola parts and removed the accessible detector components. With the rest of the recovery team and the Basler flight crew on site, we had sufficient man power and rigging equipment to move the large Cherenkov detectors safely into the aircraft. Given the advanced stage of the disassembly, the decision was made to use the 5 hours of Basler ground time and load all detector components along with the SuperTIGER recovery team into a single Basler flight back to South Pole Station. There the cargo was repalletized for an LC-130 flight to McMurdo, where the instrument was packed into a sea container for the vessel shipment to the continental US. The instrument was received at NASA Goddard Space 
Flight Center on April 2015 and is presently being evaluated and refurbished for a future flight of SuperTIGER.

\subsection{Charge Identification}

The data analysis of SuperTIGER employs two complementary techniques to assign nuclear charge, $Z$, to incident particles. For energies above the $\mathrm{C} 1$ threshold $(\sim 0.3 \mathrm{GeV} /$ nucleon) but below the $\mathrm{C} 0$ threshold $(2.5 \mathrm{GeV} /$ nucleon or $3.3 \mathrm{GeV} /$ nucleon, depending on the aerogel refractive-index in the hit half-module), the charge is determined from the signals of the upper two scintillation counters $(\mathrm{S} 1+\mathrm{S} 2)$ and the acrylic $(\mathrm{C} 1)$ Cherenkov counter (Below $\mathrm{C} 0)$. For energies greater than the $\mathrm{C} 0$ threshold charge is derived from the acrylic $(\mathrm{C} 1)$ and the aerogel $(\mathrm{C} 0)$ Cherenkov counters (Above $\mathrm{C} 0$ ). A consistency cut of the charge derived from the upper (S1+S2) and bottom (S3) scintillation counters can be used to reject charge changes due to interactions in the instrument. Presently, our analysis has focused on the available TDRSS data and LOS data. The analysis only contains events whose telemetry packages passed a checksum test. With the recently recovered on-board data storage, we plan to extend our analysis to the entire data set.

Before deriving a charge from the PMT signals of the scintillation and Cherenkov counters, each PMT signal was pedestal and gain corrected. The pedestal was determined for successive 20-minute interval of flight and the PMT gain correction was obtained using the ${ }_{26} \mathrm{Fe}$ peak for successive 2-hour intervals. The position-dependent response (mapping) for each detector was produced on a $2 \mathrm{~cm} \times 2 \mathrm{~cm}$ grid using Fe events from the full flight duration, here the position and incident angle are determined by the fiber hodoscope layers. Details of the data analysis can be found in these proceedings[28].

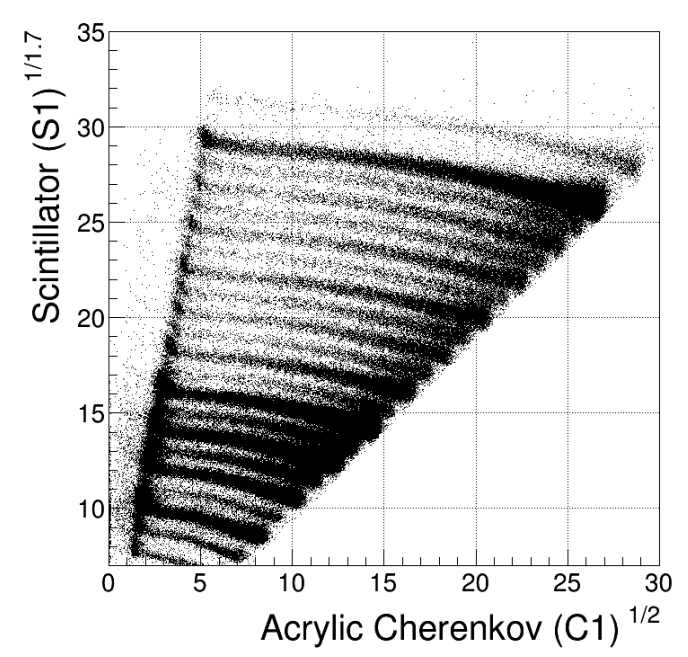

Figure 5: Cross-plots of SuperTIGER data for scintillation detector (S1) versus acrylic Cherenkov (C1).

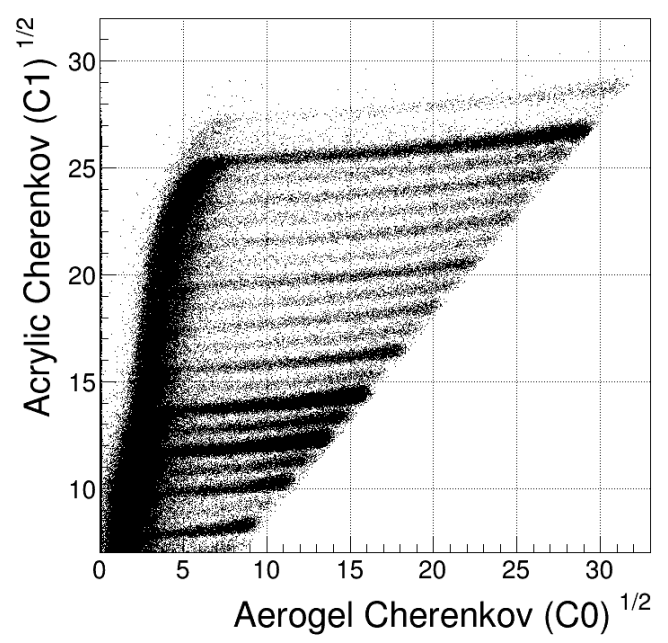

Figure 6: Cross-plots of SuperTIGER data for acrylic Cherenkov (C1) versus aerogel Cherenkov (C0).

With the above mentioned corrections applied, the incident charge, $Z$, is determined for below $\mathrm{C} 0$ events with the combined signal of the upper scintillation counters $(\mathrm{S} 1+\mathrm{S} 2)$ and the acrylic 
Cherenkov counter (C1). The energy loss $d E / d x$ in the scintillation counter follows $Z^{2}$. However, with increasing charge the energy deposited in a small volume will reach a saturation point above which the organic scintillator will not have a proportional light yield. Empirically the scintillator saturation can be approximated as $d L / d x \propto Z^{1.7}$ for lower charges[29]. Figure 5 shows a cross plot for Below $\mathrm{C} 0$ events of the scintillator $\left(\mathrm{S}^{1 / 1.7}\right)$ versus the acrylic Cherenkov $\left(\mathrm{C} 1^{1 / 2}\right)$ response, providing a quasi-charge scale for both axes. The charge bands up to ${ }_{28} \mathrm{Ni}$ are clearly visible and can be described by the scintillator signal and a small velocity correction from the acrylic Cherenkov counter. For elements above ${ }_{28} \mathrm{Ni}$ a model prediction is needed to establish charge bands. We found that the Voltz et al.[30] model discribes the SuperTIGER data best and it has been used to establish charge bands for the Below $\mathrm{C} 0$ events (see [31]). For the Above $\mathrm{C} 0$ events both Cherenkov counters are used to determine charge. Here the charge is determined from $\mathrm{C} 1$ with a small velocity correction from $\mathrm{C} 0$. Figure 6 shows a cross plot for Above $\mathrm{C} 0$ events where the acrylic Cherenkov $\left(\mathrm{C}^{1 / 2}\right)$ is plotted versus the aerogel Cherenkov $\left(\mathrm{C}^{1 / 2}\right)$ response. As detailed elsewhere in these proceedings[31] the charge bands established for the above and below $\mathrm{C} 0$ events can be used to generate charge histograms.

\subsection{Abundance of Ultra-Heavy Elements for SuperTIGER}

To determine the elemental abundances of the ultra-heavy GCR, the detected charges, Z, determined in the two energy bands (Below $\mathrm{C} 0$ and Above $\mathrm{C} 0$ ), are combined into a histogram as shown in Figures 7 and 8. For ${ }_{26} \mathrm{Fe}$ we achieved a charge resolution of 0.16 c.u. For charges $Z \leq 32$, the charge distribution was reproduced with a multi-peaked gaussian fitting routine initially developed for ACE/CRIS and later adapted for the TIGER analysis[32]. For charges with $Z>32$, the elemental abundance was determined by simply counting the number of events in each well-defined peak as shown in Fig. 8. For $Z>29$ we used a less restrictive cut to increase statistics.

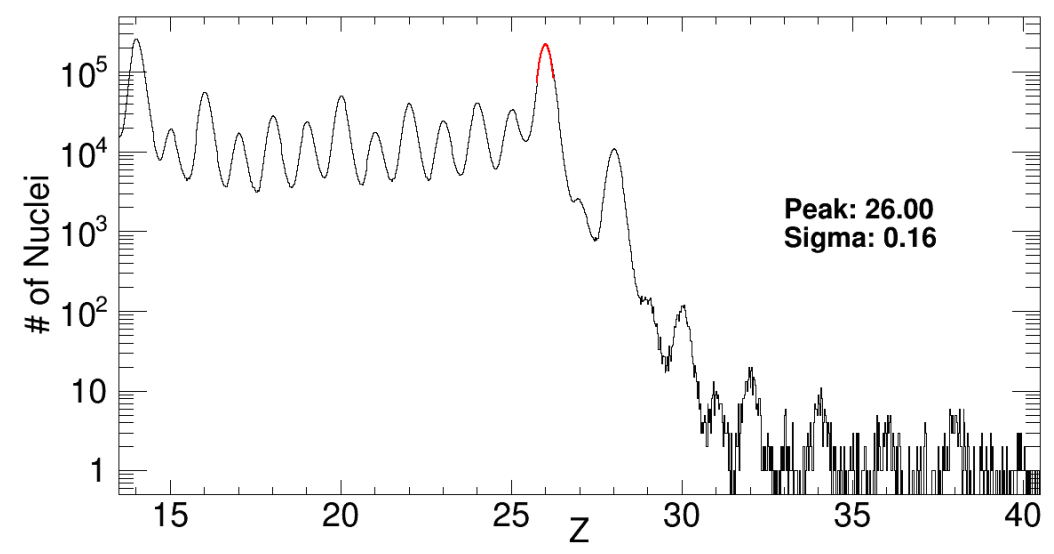

Figure 7: Charge histogram (0.025 c.u. binning) for above and below C0 SuperTIGER data stating the ${ }^{26} \mathrm{Fe}$ peak location and resolution.

The detected number of nuclei within the SuperTIGER instrument needs to be corrected for charge-dependent interaction losses in the instrument to derive a top-of-instrument (TOI) value. From the TOI abundance the top-of-atmosphere value (TOA) is calculated, by accounting for 
charge-dependent energy losses as well as nuclear interactions in the atmosphere. For the interactions within the SuperTIGER instrument we used the interaction mean free path for each $Z$ $[33,34]$, and the appropriate path length within the instrument for each material used in the detector stack. Our data selection cuts eliminate charge-changing interactions in the instrument and with this instrumental correction applied we obtain the TOI abundance.

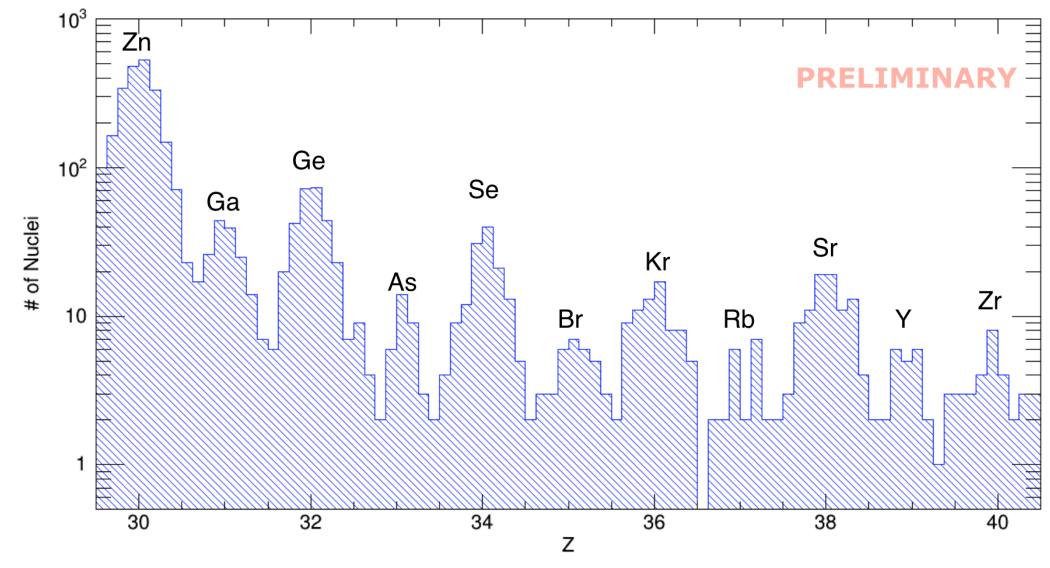

Figure 8: Ultra-Heavy Charge Histogram (binning 0.125 c.u.).

The correction for the energy losses as well as nuclear interactions in the atmospheric overburden uses the same method as in TIGER (see [1]) and the results of the corrected TOA abundances (normalized to ${ }_{26} \mathrm{Fe}=1$ ) are shown in Fig. 9 together with results from ACE/CRIS[11] and HEAO-3-C2 [35]. The results are compared to Solar System abundances from Lodders[22]. The SuperTIGER data points are presently statistical error bars only. The SuperTIGER results are in agreement with the other measurements, but have improved statistics.

We are currently working on obtaining source abundances using our measured TOI abundances and a cosmic ray propagation code[36]. To obtain cosmic-ray abundances at the source we used the same propagation parameters as were obtained for the TIGER data, discussed in [1]. Figure 10 shows the preliminary GCR source abundance for SuperTIGER (statistical uncertainties only) compared to a mix of material with $80 \%$ of Solar System abundances and $20 \%$ massive star outflow and ejecta. Also shown are the TIGER and ACE/CRIS points. With the SuperTIGER data the mass-dependent ordering of both the volatile and refractory elements remains, with reduced error bars for higher charges. We are presently investigating the systematic uncertainties.

\section{Recent Results from ACE/CRIS}

The Cosmic Ray Isotope Spectrometer (CRIS) on the NASA Advanced Composition Explorer (ACE) satellite has provided a number of important measurements of isotopic and elemental GCR composition, including UHGCR measurements. The ACE spacecraft was launched in Aug. 1997 [37] and the CRIS instrument[38] uses the $d E / d x$ vs residual Energy technique to identify incident particles. ACE/CRIS has been taking data for more than 17 years, collected $\sim 625$ nuclei with atomic number $Z \geq 30$, and shows excellent resolution with clear separation between the charges 


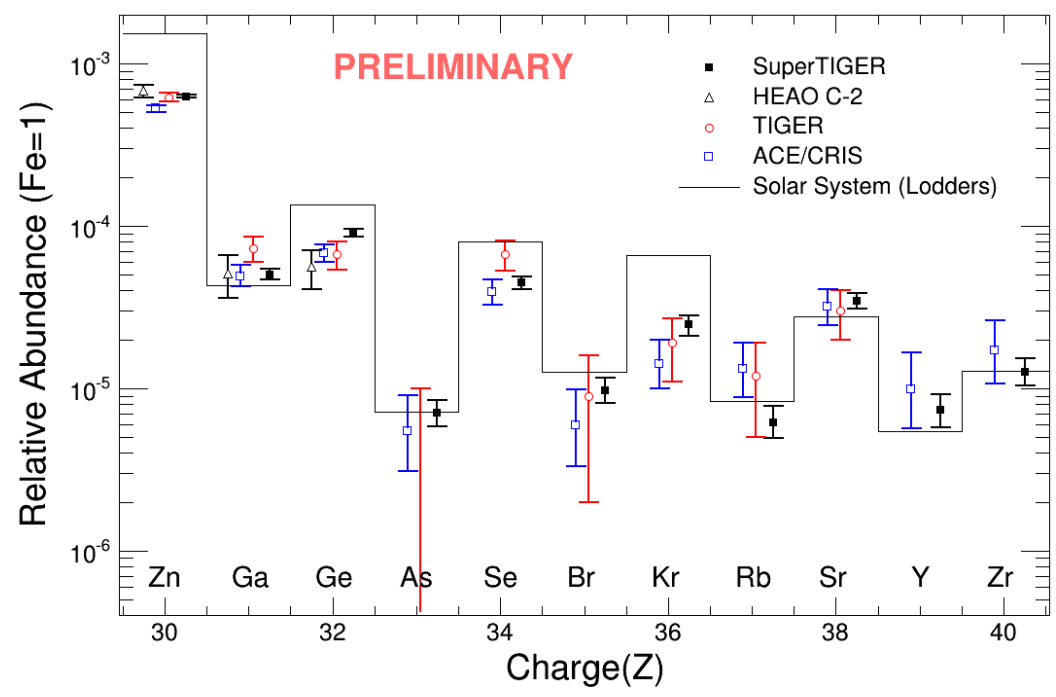

Figure 9: Comparison of SuperTIGER TOA abundances with results from ACE/CRIS[11], TIGER[1], and HEAO-C3[35]. SuperTIGER error bars are statistical only.

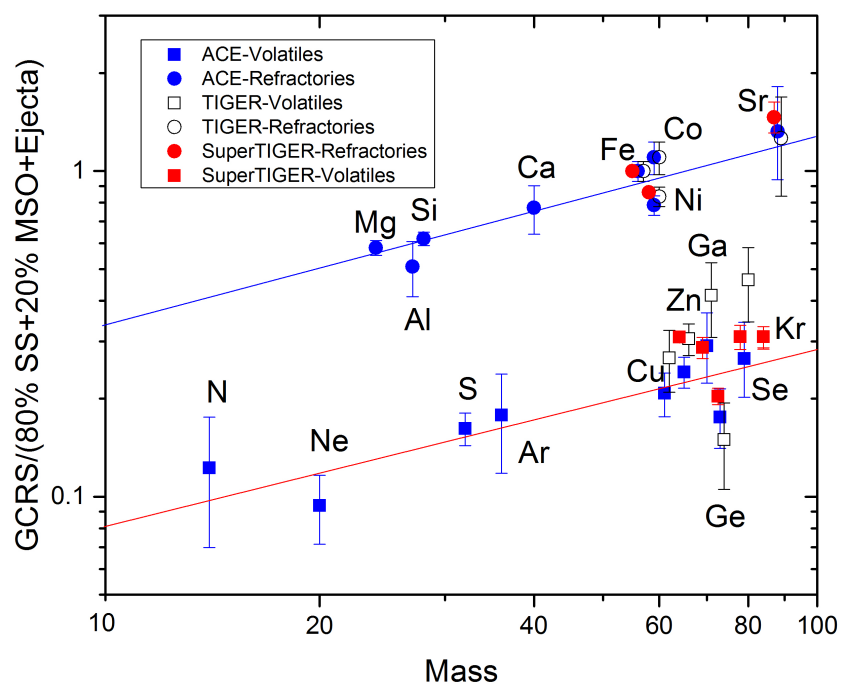

Figure 10: Comparison of the SuperTIGER refractory and volatile elements GCR source abundances with ACE/CRIS and TIGER and predicted source material. SuperTIGER error bars are statistical errors only.

for $30 \leq Z \leq 40$, see Figure 11. The CRIS data are also plotted in Figure 10 (blue data points) and show generally good agreement with SuperTIGER and TIGER results. The CRIS instrument is sensitive to nuclei in the energy range $150 \leq E \leq 600 \mathrm{MeV} /$ nucleon.

Previously, the CRIS instrument demonstrated a lack of ${ }^{59} \mathrm{Ni}$ in the GCR[39]. This isotope, present at the sources, only decays by electron capture, with a half-life of $7.4 \times 10^{5}$ years. Once orbiting electrons have been stripped off due to GCR acceleration, the isotope is essentially stable. The lack of ${ }^{59} \mathrm{Ni}$ in the GCR near Earth is evidence that at least $\sim 10^{5}$ years must have elapsed 


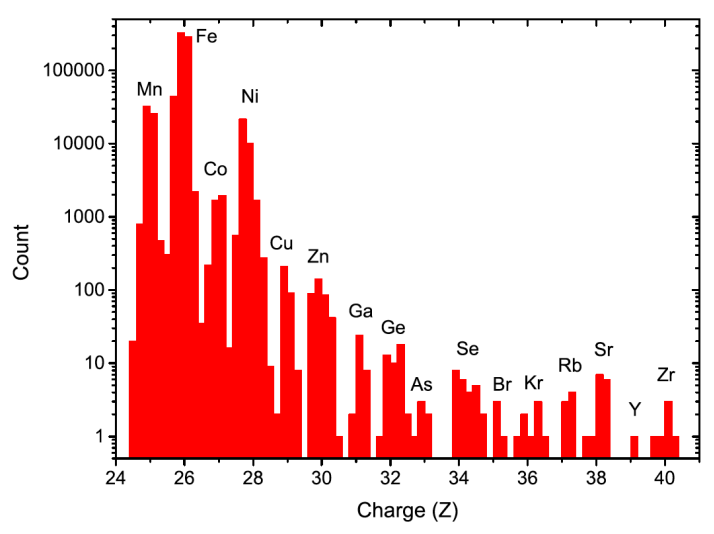

Figure 11: ACE/CRIS UHGCR Elemental Composition[11] (data 4 between December 1997 and 2 April 2011).

between nucleosynthesis and cosmic-ray acceleration.

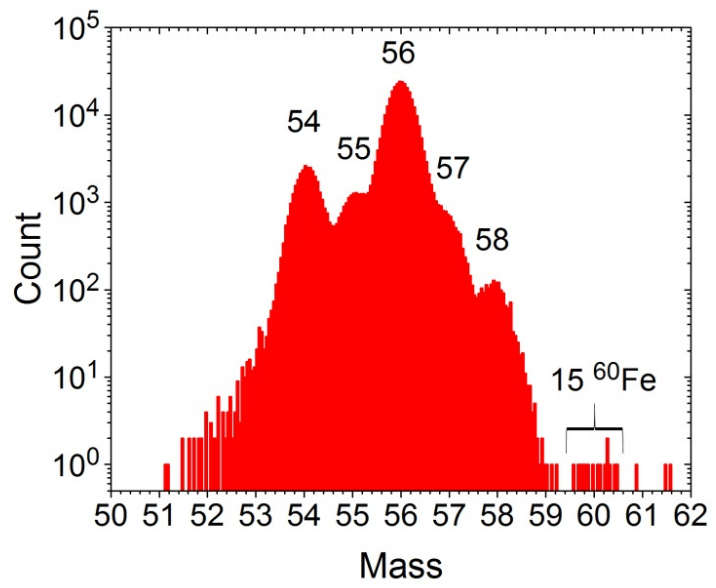

Figure 12: Mass Histogram for ${ }_{26} \mathrm{Fe}$.

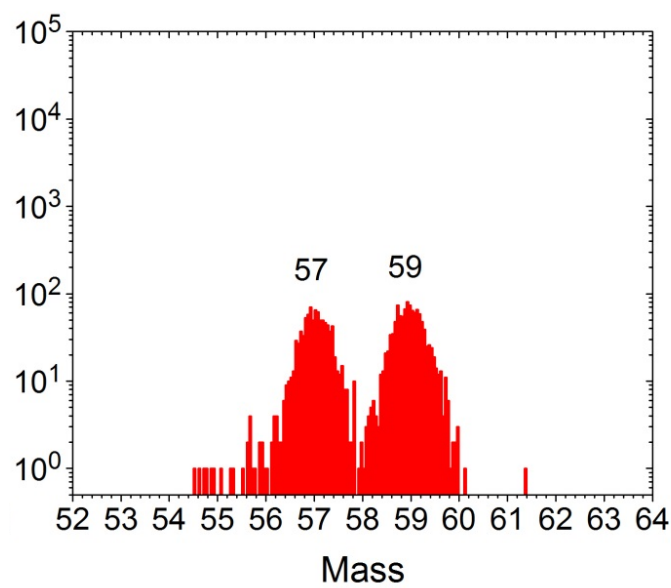

Figure 13: Mass Histogram for ${ }_{27} \mathrm{Co}$.

Recently, the CRIS team has analyzed 17 years of data to determine the isotopic composition of Fe[40]. Figure 12 shows the iron mass histogram with excellent mass resolution of $0.24 \mathrm{amu}$. Fifteen well resolved ${ }^{60} \mathrm{Fe}$ events were identified, corresponding to $2.95 \times 10^{5}{ }^{56} \mathrm{Fe}$ events in this data set. We estimate that $\sim 1$ of the ${ }^{60} \mathrm{Fe}$ could be of secondary origin, produced by nuclear interactions of heavier nuclei, primarily ${ }^{62} \mathrm{Ni}$ and ${ }^{64} \mathrm{Ni}$, in the interstellar medium. To estimate how many of the 15 events identified as ${ }^{60} \mathrm{Fe}$ might be spillover from ${ }^{58} \mathrm{Fe}$, we show the corresponding histogram of the cobalt isotopes in Figure 13. In this plot, ${ }^{59} \mathrm{Co}$ has roughly the same number of events as are in the ${ }^{58} \mathrm{Fe}$ peak. There is only 1 event near ${ }^{61} \mathrm{Co}$ that could be a high-mass spillover from ${ }^{59} \mathrm{Co}$. This is analogous to a possible spillover from ${ }^{58} \mathrm{Fe}$ to ${ }^{60} \mathrm{Fe}$. This is a strong argument that of the 15 nuclei identified as ${ }^{60} \mathrm{Fe}$ in Figure 12, only $\sim 1$ could be spillover from ${ }^{58} \mathrm{Fe}$. We conclude that 13 of the 15 nuclei identified as ${ }^{60} \mathrm{Fe}$ are primary particles. Thus we have observed $13 \pm 3.8$ (stat.) \pm 1 (syst.) $=13 \pm 4.8$ primary ${ }^{60} \mathrm{Fe}$ with $2.95 \times 10^{5}{ }^{56} \mathrm{Fe}$, giving a primary ${ }^{60} \mathrm{Fe} /{ }^{56} \mathrm{Fe}$ ratio near Earth of $(4.4 \pm 1.6) \times 10^{-5}$. This implies a ratio at the cosmic-ray acceleration source of $(0.80 \pm 0.30) \times 10^{-4}[40]$. From this we have obtained an upper limit on the time between 
nucleosynthesis and acceleration of several million years.

The fact that cosmic-ray acceleration occurs within a few million years of nucleosynthesis, but not in the same supernova where the Fe-group nuclei are synthesized requires that there are at least two nearby supernovae separated by only a few million years. This need for nearby supernovae closely spaced in time strongly suggests acceleration in associations of massive stars (OB associations). Thus our observation of ${ }^{60} \mathrm{Fe}$ gives strong support for the emerging understanding[16] that $\mathrm{OB}$ associations are the primary environment for acceleration of Galactic cosmic rays.

\section{HNX the Next Step}

SuperTIGER and ACE/CRIS are complementary instruments for understanding the origin of UHGCR, covering energy ranges of $E>0.8 \mathrm{GeV} /$ nucleon and $150 \leq E \leq 600 \mathrm{MeV} /$ nucleon, respectively. Both instruments have comparable exposure factors of $\sim 1$ and $\sim 0.5 \mathrm{~m}^{2} \mathrm{sr}$ yr, respectively, for a single 44-day SuperTIGER balloon flight and 18 years of CRIS, but to extend the measurements to higher charges, including actinides, far larger exposure factors are needed. This is the motivation for the Heavy Nuclei eXplorer (HNX) mission.

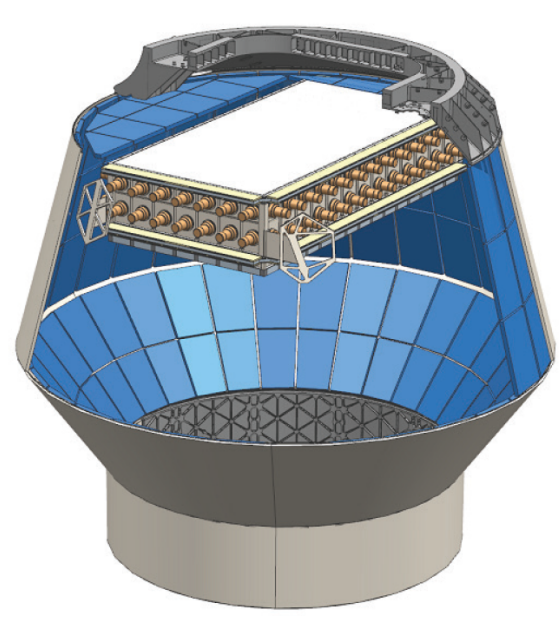

Figure 14: Cutaway view of HNX in the DragonLab capsule. The ECCO glass modules cover the walls and CosmicTIGER is located at the top.

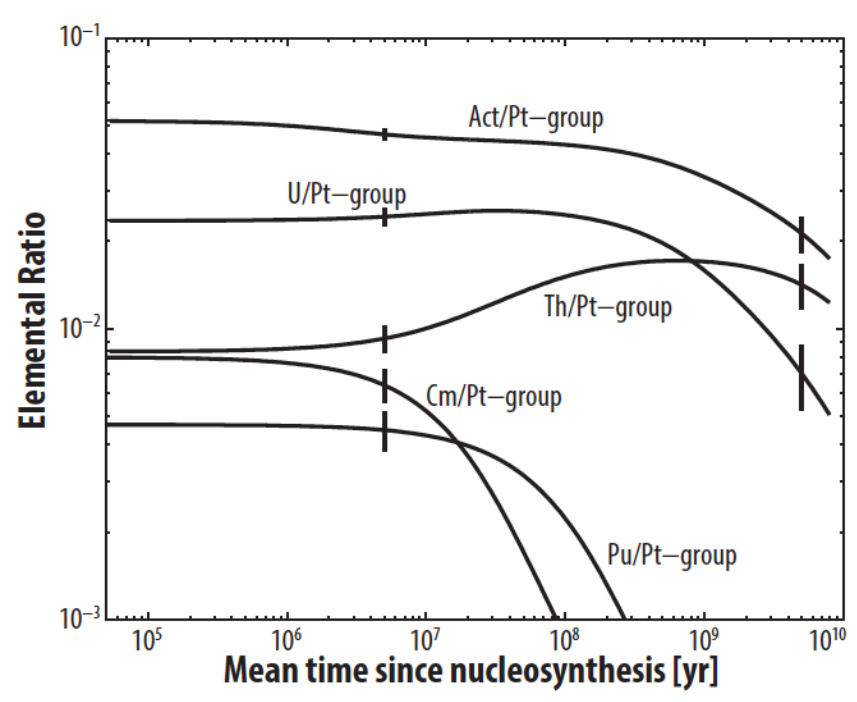

Figure 15: Relative actinide daughter-parent ratios and their evolution as a function of GCR age. Error bars are for a $2 \mathrm{yr}$ HNX mission.

HNX is a new mission in the NASA Small Explorer class and is a collaboration of NASA Goddard Space Flight Center, University of California, Berkeley, Washington University in St. Louis, and the Jet Propulsion Laboratory. HNX will investigate the nature of the reservoirs of nuclei at the cosmic-ray sources, the mechanisms by which nuclei are injected into the cosmic accelerators, and the acceleration mechanism. HNX will measure, for the first time, the abundance of every individual element in the periodic table from carbon through the actinides, providing the first measurement of many of these elements. Several tens of thousands of UHGCR nuclei with atomic number $Z \geq 30$ will be recorded, including about 50 actinides. 
To measure UHGCR with unprecedented statistics and individual element resolution over its full measurement range, HNX will use two large instruments, the Extremely-heavy Cosmic-ray Composition Observer (ECCO) using $21 \mathrm{~m}^{2}$ of sophisticated BP-1 barium phosphate glass tracketch detectors and the Cosmic-ray Trans-Iron Galactic Element Recorder (CosmicTIGER) using a $2 \mathrm{~m}^{2}$ suite of electronic detectors evolved from SuperTIGER. Compared to the exposure that could be achieved by SuperTIGER in a future LDB flight with a typical duration of 30 days, in a nominal 2-year HNX mission the exposure of the ECCO instrument will be approximately two orders of magnitude greater $\left(97 \mathrm{~m}^{2} \mathrm{sr} \mathrm{yr}\right)$ and the CosmicTIGER exposure will be about an order of magnitude greater $\left(8.4 \mathrm{~m}^{2} \mathrm{sr} \mathrm{yr}\right)$. HNX will be accommodated in the SpaceX DragonLab orbiting laboratory that will also return it to Earth for post-flight processing of the ECCO detectors. Figure 14 shows a cutaway view of the spacecraft with ECCO and CosmicTIGER installed.

HNX will make the first measurement of all elements from ${ }_{6} \mathrm{C}$ through the remainder of the periodic table in a single mission. ECCO will measure individual abundances of elements with $Z \geq 70$ and kinetic energy $\geq 1 \mathrm{GeV} /$ nucleon. CosmicTIGER will cover the charge range of $6 \leq$ $Z \leq 96$ and $E>0.2 \mathrm{GeV} /$ nucleon and measure the energy spectra of the more common elements up to about $10 \mathrm{GeV} /$ nucleon.

HNX measurements will determine whether GCR are accelerated from new or old material, and find their age. The measured elemental abundances will enable us to determine the mix of r-and s-process material responsible for the UHGCRs and investigate how UHGCR elements are selected for acceleration. HNX will enable us to measure refractory and volatile elements with greatly extended mass range and far higher statistics, thereby further defining the mechanism by which nuclei are selected for acceleration and the mass dependence of UHGCR acceleration. The measurement of the actinides $\left({ }_{90} \mathrm{Th},{ }_{92} \mathrm{U},{ }_{94} \mathrm{Pu},{ }_{96} \mathrm{Cm}\right.$ ) is a sensitive test of the absolute UHGCR age, since their half-lives span the time-scale of galactic chemical evolution.

The relative abundances of the UHGCR strongly depend on the age of the GCR source material. Of particular importance are the daughter-parent ratios such as $\mathrm{Th} / \mathrm{U}$ and $(\mathrm{Th}, \mathrm{U}, \mathrm{Pu}) / \mathrm{Cm}$. Figure 15 illustrates how the age of the GCR is constrained by the elemental ratios of the actinides. As an example, if the GCR source material was synthesized recently, for example within the characteristic $\sim 10 \mathrm{Myr}$ age of an OB association, then the observed Plutonium ${ }_{94} \mathrm{Pu}$ ) and Curium (96 $\mathrm{Cm}$ ) abundances should be much higher than if the GCR source material is old ISM. HNX has a sufficient exposure factor to detect significant numbers of ${ }_{94} \mathrm{Pu}$ and ${ }_{96} \mathrm{Cm}$ nuclei if their GCR sources have a recently synthesized component as suggested by the SuperTIGER and ACE/CRIS data.

\section{Conclusions}

The SuperTIGER instrument was developed to precisely measure the abundances of the ultraheavy galactic cosmic rays, with single-element resolution and high statistics. In its first 55-day balloon flight the instrument operated nominally and returned excellent data collecting over $4 \times 10^{6}$ Iron nuclei, over 7 times more than in the 2001 and 2003 TIGER flights combined. The data analysis for the first SuperTIGER flight is being finalized and will provide a further test of the OB associations as the origin of Galactic cosmic rays. The present results of the SuperTIGER analysis 
show that it is in good agreement with satellite measurements (including ACE/CRIS), but has better statistics. Currently, preparations are underway to refly SuperTIGER to further improve statistics.

The recent ACE/CRIS detection of ${ }^{60} \mathrm{Fe}$ in the GCR near Earth provides an upper limit on the elapsed time of $<10 \mathrm{Myr}$ between nucleosynthesis and cosmic-ray acceleration. Earlier ACE/CRIS results found an absence of ${ }^{59} \mathrm{Ni}$ in the GCR near Earth, setting a lower limit of that elapsed time of greater than $0.1 \mathrm{Myr}$, leading to the conclusion that the supernova in which the nucleosynthesis occurs cannot be the supernova whose shock wave accelerates the material to cosmic-ray energies. Thus, the observation of ${ }^{60} \mathrm{Fe}$ gives strong support for the model in which OB associations are the primary environment for acceleration of Galactic cosmic rays.

The next step in the answering the question of the origin of GCR will be the HNX mission, which will provide excellent charge resolution and large exposure to measure the abundances of individual elements with unprecedented statistics from carbon through the actinides in a single mission. HNX will provide the crucial elemental abundance measurements required to fully understand the origin, acceleration, and subsequent history of UHGCR nuclei as well as the mix of nucleosynthesis processes responsible for their production. HNX will definitively determine the age for GCR by precisely measuring the relative abundances of the actinides with high accuracy.

Acknowledgment: SuperTIGER is supported by NASA under the ROSES 2007 APRA program under grants NNX09AC17G to Washington University in St. Louis and NNX09AC18G to Caltech and JPL, and APRA07-0146 to NASA/GSFC. We wish to thank the Columbia Scientific Balloon Facility (CSBF) personnel and the Wallops Balloon Program Office (BPO) for their excellent efforts that resulted in our highly successful long-duration balloon flight. We also wish to thank the National Science Foundation Office of Polar Programs for their outstanding logistical support which made this investigation possible. The ACE data analysis is supported by NASA Grant NNX13AH66G.

\section{References}

[1] B. F. Rauch et al., Cosmic Ray origin in OB Associations and Preferential Acceleration of Refractory Elements: Evidence from Abundances of Elements ${ }_{26} \mathrm{Fe}$ through ${ }_{34} \mathrm{Se}$, ApJ 697 (June, 2009) 2083-2088, [arXiv:0906.2021].

[2] M. Cassé and P. Goret, Ionization models of cosmic ray sources, ApJ 221 (Apr., 1978) 703-712.

[3] J.-P. Meyer, Solar-stellar outer atmospheres and energetic particles, and galactic cosmic rays, ApJS 57 (Jan., 1985) 173-204.

[4] J. T. Schmelz, D. V. Reames, R. von Steiger, and S. Basu, Composition of the Solar Corona, Solar Wind, and Solar Energetic Particles, ApJ 755 (Aug., 2012) 33.

[5] W. R. Binns et al., Abundances of ultraheavy elements in the cosmic radiation - Results from HEAO 3, ApJ 346 (Nov., 1989) 997-1009.

[6] R. I. Epstein, The acceleration of interstellar grains and the composition of the cosmic rays, MNRAS 193 (Dec., 1980) 723-729.

[7] J.-P. Bibring and C. J. Cesarsky, Interstellar grains as seeds for galactic cosmic rays, International Cosmic Ray Conference 2 (1981) 289-291. 
[8] J.-P. Meyer, L. O. Drury, and D. C. Ellison, Galactic Cosmic Rays from Supernova Remnants. I. A Cosmic-Ray Composition Controlled by Volatility and Mass-to-Charge Ratio, ApJ 487 (Sept., 1997) 182-196, [astro-ph/9704267].

[9] D. C. Ellison, L. O. Drury, and J.-P. Meyer, Galactic Cosmic Rays from Supernova Remnants. II. Shock Acceleration of Gas and Dust, ApJ 487 (Sept., 1997) 197-217, [a stro-ph/ 9704293 ].

[10] A. J. Westphal et al., Evidence against stellar chromospheric origin of Galactic cosmic rays, Nature 396 (Nov., 1998) 50-52.

[11] W. R. Binns et al., Constraints on Galactic Cosmic-Ray Origins from Elemental and Isotopic Composition Measurements, in 33rd International Cosmic Ray Conf. (Rio de Janeiro) 0646 (2013).

[12] H. S. Ahn et al., Measurements of the Relative Abundances of High-energy Cosmic-ray Nuclei in the TeV/Nucleon Region, ApJ 715 (June, 2010) 1400-1407.

[13] S. E. Woosley and A. Heger, Nucleosynthesis and remnants in massive stars of solar metallicity, Phys. Rep. 442 (Apr., 2007) 269-283, [astro-ph/0702176].

[14] T. Montmerle, On gamma-ray sources, supernova remnants, OB associations, and the origin of cosmic rays, ApJ 231 (July, 1979) 95-110.

[15] C. J. Cesarsky and T. M. Montmerle, Cosmic rays from OB associations and supernovae Anti-protons and the origin of local cosmic rays, International Cosmic Ray Conference 9 (1981) 207-210.

[16] J. C. Higdon, R. E. Lingenfelter, and R. Ramaty, Cosmic-Ray Acceleration from Supernova Ejecta in Superbubbles, ApJ 509 (Dec., 1998) L33-L36.

[17] J. C. Higdon and R. E. Lingenfelter, The Superbubble Origin of ${ }^{22} \mathrm{Ne}$ in Cosmic Rays, ApJ 590 (June, 2003) 822-832.

[18] J. C. Higdon and R. E. Lingenfelter, OB Associations, Supernova-generated Superbubbles, and the Source of Cosmic Rays, ApJ 628 (Aug., 2005) 738-749.

[19] J. C. Higdon and R. E. Lingenfelter, The Galactic Spatial Distribution of OB Associations and their Surrounding Supernova-generated Superbubbles, ApJ 775 (Oct., 2013) 110, [arXiv : 1302 . 1223].

[20] E. Parizot, A. Marcowith, E. van der Swaluw, A. M. Bykov, and V. Tatischeff, Superbubbles and energetic particles in the Galaxy. I. Collective effects of particle acceleration, A\&A $\mathbf{4 2 4}$ (Sept., 2004) 747-760, [astro-ph/0405531].

[21] K. Reitberger, R. Kissmann, A. Reimer, O. Reimer, and G. Dubus, High-energy Particle Transport in Three-dimensional Hydrodynamic Models of Colliding-wind Binaries, ApJ 782 (Feb., 2014) 96, [arXiv:1401.1323].

[22] K. Lodders, Solar System Abundances and Condensation Temperatures of the Elements, ApJ 591 (July, 2003) 1220-1247.

[23] W. R. Binns et al., Cosmic-Ray Neon, Wolf-Rayet Stars, and the Superbubble Origin of Galactic Cosmic Rays, ApJ 634 (Nov., 2005) 351-364, [astro-ph/ 0508398 ].

[24] N. Prantzos, On the origin and composition of Galactic cosmic rays, A\&A 538 (Feb., 2012) A80, [arXiv:1112.4343].

[25] S. Heinz and R. Sunyaev, Cosmic rays from microquasars: A narrow component to the CR spectrum?, A\&A 390 (Aug., 2002) 751-766, [a stro-ph/ 0204183 ]. 
[26] S. Geier et al., A search for the signature of microquasars in the cosmic ray iron spectrum measured by TIGER, Advances in Space Research 37 (2006) 1955-1959.

[27] W. R. Binns et al., The SuperTIGER Instrument: Measurement of Elemental Abundances of Ultra-Heavy Galactic Cosmic Rays, ApJ 788 (June, 2014) 18-28.

[28] M. Sasaki et al., SuperTIGER and the Origin of Galactic Cosmic-Rays, in Proceedings of ICRC2015 (2015).

[29] J. T. Link, Measurements of ultra-heavy galactic cosmic rays with the TIGER instrument. PhD thesis, Washington University in St. Louis, 2003.

[30] R. Voltz, J. L. da Silva, G. Laustriat, and A. Coche, Influence of the Nature of Ionizing Particles on the Specific Luminescence of Organic Scintillators, The Journal of Chemical Physics 45 (Nov., 1966) 3306-3311.

[31] R. P. Murphy et al., Abundances of Ultra-Heavy Galactic Cosmic Rays from the SuperTIGER Instrument, in Proceedings of ICRC2015 (2015).

[32] L. M. Scott, Cosmic-ray energy loss in the heliosphere and interstellar reacceleration. PhD thesis, Washington University, Missouri, USA, Nov., 2005.

[33] B. S. Nilsen et al., Fragmentation cross sections of relativistic ${ }^{84}{ }_{36} \mathrm{Kr}$ and ${ }^{109}{ }_{47} \mathrm{Ag}$ nuclei in targets from hydrogen to lead, Phys Rev C 52 (Dec., 1995) 3277-3290.

[34] G. D. Westfall et al., Fragmentation of relativistic ${ }^{56}$ Fe, Phys Rev C 19 (Apr., 1979) 1309-1323.

[35] B. Byrnak et al., The Abundances of the Elements with $Z<26$ IN the Cosmic Radiation, International Cosmic Ray Conference 2 (Aug., 1983) 29.

[36] M. E. Wiedenbeck et al., An Overview of the Origin of Galactic Cosmic Rays as Inferred from Observations of Heavy Ion Composition and Spectra, Spac Sci Rev 130 (June, 2007) 415-429.

[37] E. C. Stone et al., The Advanced Composition Explorer, Space Seince Review 86 (July, 1998) 1-22.

[38] E. C. Stone et al., The Cosmic-Ray Isotope Spectrometer for the Advanced Composition Explorer, Space Science Reviews 86 (July, 1998) 285-356.

[39] M. E. Wiedenbeck et al., Constraints on the Time Delay between Nucleosynthesis and Cosmic-Ray Acceleration from Observations of ${ }^{59} \mathrm{Ni}$ and ${ }^{59} \mathrm{Co}$, ApJl 523 (Sept., 1999) L61-L64.

[40] M. H. Israel et al., Observation of ${ }^{60} \mathrm{Fe}$ in the Galactic Cosmic Rays, in Proceedings of ICRC2015 (2015). 